\title{
Polio vaccine: is it time for a change?
}

\author{
Adam Finn, Frank Bell
}

It is hoped, and widely expected, that early in the next century the wild-type polio virus will be declared extinct as a result of an eradication programme started in 1988 by the World Health Assembly using live attenuated trivalent oral (Sabin) polio vaccine (OPV). ${ }^{1}$ This potential achievement is reminiscent of the successful eradication of smallpox in 1977, but there are important differences. Most polio infections are subclinical and those that cause myelitis are not reliably distinguished from other causes of acute flaccid paralysis on clinical grounds alone. Highly effective clinical and laboratory surveillance will be needed to ensure that polio has really gone. ${ }^{2}$ Most importantly in practical terms, worldwide immunisation will be necessary until global eradication is certified to prevent re-importation and outbreaks in children who have not been immunised. This is confirmed by recent outbreaks in areas where immunisation rates with OPV have fallen, or where pockets of unimmunised subjects have developed-for example, in Albania $^{3}$ and Chechnya. ${ }^{4}$ In contrast, during the final years before smallpox eradication, a number of developed countries stopped routine immunisation as it was causing significant morbidity and mortality in the absence of any cases of smallpox, so that the risk:benefit ratio was against continuing. Unlike polio, the importation and spread of the disease was judged to be unlikely to occur and easy to control.

A similar risk:benefit problem now faces countries that have achieved effective control of polio using OPV. Almost all of the small residual number of cases of paralytic polio in these countries are caused by the vaccine virus, ${ }^{5}{ }^{6}$ either in recipients of the vaccine (usually children) or in non-immune subjects in contact with recipients to whom the virus is spread (usually adults). Vaccine associated paralytic polio (VAPP) occurs as a consequence of the reversal of attenuating mutations in the vaccine virus-events that occur extremely frequently, ${ }^{78}$ but only rarely with adverse consequences in immunologically normal subjects. The overall incidence of VAPP (defined as cases of permanent paralysis) is estimated to be one in 400000 to one in 520000 first doses, and one in 12.3 million subsequent doses. ${ }^{910}$ A minority of cases of VAPP are caused by humoral or combined immunodeficiency in the recipient. ${ }^{5}$ Of 28 confirmed cases of polio in the UK between 1985 and 1994, 19 were VAPP, six were acquired abroad, and three were of unknown origin. ${ }^{6}$

As immunisation cannot stop at present, there would be no dilemma about VAPP except that an alternative highly effective vaccine is now available: enhanced potency trivalent inactivated (Salk) polio vaccine or eIPV. Inactivated vaccine has been used successfully to control polio in several Scandinavian countries and in the Netherlands since the 1950s. This vaccine does not cause VAPP as cases are not reported in these countries. So although the widespread use of OPV is being promoted vigorously in countries in which polio is, or was until recently, endemic, its continued use is being questioned in countries where indigenous polio has disappeared, just as with the smallpox vaccine in the 1970s. Criteria have been drawn up, which countries considering the change should fulfil and include the absence of indigenous poliomyelitis for at least five years and reliable vaccine coverage above $90 \%$.

Debate about the relative merits of these two vaccines is not new. It was conducted with vigour by their inventors Salk and Sabin from the 1960 s until their deaths, ${ }^{112}$ and is continued by many others. ${ }^{13}{ }^{14}$ Many countries have used the two vaccines at different times and in different ways over the past 40 years. France has moved progressively away from OPV since the early 1980 s. $^{15}$ More recently, Canada has almost entirely switched to IPV and Germany is now set to do the same.

A third alternative to the exclusive use of either OPV or IPV is to adopt a schedule that involves the two vaccines. There is extensive experience of this approach in Denmark and Israel. ${ }^{16}$ Current combined schedules usually start with two doses of IPV in infancy followed by OPV boosters in the second year and preschool. The logic for the approach is that the use of IPV should reduce the incidence of VAPP, which most commonly occurs after the first dose of OPV, by inducing sufficient serum neutralising antibody to prevent invasive infection on subsequent exposure to OPV. At the same time the schedule is postulated to retain, to some extent, the perceived benefits for continued use of OPV and seems to be at least as immunogenic as conventional schedules. ${ }^{17} 18$

Austria and Italy have opted for combined schedules. In the USA, where the matter has been under vigorous debate for several years, and where approximately five to 10 cases of VAPP each year have been notified and confirmed in recent years, ${ }^{5} 1920$ advisory bodies have endorsed a move towards the use of IPV, while leaving open to parents and doctors the options of using either OPV alone or IPV alone. ${ }^{21}{ }^{22}$ The recommendation for the combined schedule is based on an estimated prediction of a $50 \%$ consequential reduction in the incidence of VAPP. ${ }^{23}$ Some cases will continue to occur as long as OPV is in use, however, and these moves are likely to precede a complete switch to IPV in the USA in due course. 
Where does this leave countries like the UK, Spain, and Australia, where OPV continues to be used exclusively and where indigenous wild polio transmission has long stopped? Aside from a reluctance to rock the boat, not in itself a valid argument, the three most commonly voiced arguments for maintenance of the status quo $^{13}$ are: OPV induces superior population wide protection against imported infection (as opposed to disease) because of the induction of mucosal immunity; OPV induces a broader population wide immunity from secondary spread to contacts of recipients; and IPV or a mixed schedule would cost more.

Although inferior to OPV, intestinal immune responses are induced by eIPV and reduce faecal shedding after challenge, whereas pharyngeal immune responses are approximately equivalent for the two vaccines. ${ }^{24}$ It is clear that eIPV has a major impact on the circulation of polio virus, as well as inducing individual protection against paralytic disease. ${ }^{25}$ Nevertheless, wild polio has caused disease in communities immunised with either inactivated, oral, or a combination of the two types of vaccine. ${ }^{4}$ An outbreak of nine cases occurred in Finland in 1984 in a population immunised with an IPV subsequently shown to have poor immunogenicity. ${ }^{26}$ In another outbreak in Israel, a cluster of cases of paralysis with type 1 polio virus occurred in a population in whom children under the age of 6 years had received IPV only. All but one case, however, occurred in subjects who had received an OPV containing a lower titre of type 1 vaccine than that in current use. ${ }^{27}$ Experience in Dutch and Swedish outbreaks of paralytic polio in religious sects who refuse all immunisations has shown that, in the presence of immunisation rates similar to those achieved in the UK, the spread of imported wild-type polio virus infection into an eIPV immunised population does not occur. ${ }^{28-30}$

Data suggest that relatively little secondary spread of OPV occurs in highly immunised populations and most of the secondary spread that occurs in unvaccinated groups is type 2, whose wild-type counterpart is the least virulent of the three polio types. ${ }^{31}$ Where VAPP is the prevalent type of poliomyelitis, the secondary spread of (potentially disattenuated) OPV is not only unnecessary, but actually undesirable.

Enhanced IPV is manufactured in Europe by Pasteur Merieux and by RIVM in the Netherlands. Other manufacturers are also developing the vaccine, which should ensure adequate competition and supply. Manufacturers may consider it practical to ensure that the overall costs entailed in the purchase and delivery of IPV in combination with the other antigens of primary schedule are comparable with those using OPV.

If certification of polio eradication is achieved by $2007-10$ years from writing this paper-which seems possible, so that immunisation can cease, we might predict, at current rates, 15 more cases of VAPP to be notified in the UK if we continue to use only OPV. Change to a combined schedule, at worst, might prevent half of these, perhaps only six if we take two years to decide and implement the change. Is it worth it for six cases? Certainly, if the prevention of VAPP is the object of making a change, it seems illogical to opt for a combined schedule that will only partly achieve this aim while making life substantially more complicated, all to continue using OPV for reasons that no longer seem secure. In addition, it is now widely accepted that current attempts at surveillance and reporting of acute flaccid paralysis in the UK and elsewhere in Europe are inadequate. ${ }^{6}$ We do not currently achieve WHO surveillance criteria that call for minimum reporting rates of one case of acute flaccid paralysis per 100000 children under 15 years old, having managed a rate of only 0.38 cases per year between 1991 and $1994 .^{6}$ Although some true polio cases eventually come to light long after they occur, this nevertheless means that current UK figures for VAPP incidence are probably underestimates. Perhaps we are simply no longer very good at diagnosing this rare disease. Perhaps we are calling it Guillain-Barré syndrome and not thinking to report it, or, perhaps, in young infants whose gross motor skills are still limited, we do not recognise it at all. Whatever the reason, there may be more VAPP to prevent in the next 10 years than we think. It is clear from US experience that VAPP is associated with severe permanent paralysis and occasional deaths, just like the wild-type infection. ${ }^{5}$ In addition to wishing to avoid these cases on humanitarian grounds, it may also be worth considering the potential impact on the immunisation programme in general if such cases, when they occur, generate the kind of media attention and litigation that pertussis immunisation did 20 years ago. It would certainly be better to change in anticipation of, rather than in response to, a drop in public confidence in OPV.

A change to IPV in the UK would create some practical problems. Our current first three immunisation doses at 2, 3, and 4 months begin sooner and are more closely spaced than in most other countries. France uses IPV and the same infant schedule, but gives a booster dose in the second year. There is evidence that immune responses to IPV are improved if the doses are given two months rather than one month apart. ${ }^{32} 33$ There is also data suggesting that simultaneous immunisation with IPV and pertussis vaccines may result in reduced antibody responses to pertussis. ${ }^{34}{ }^{35}$ It is likely that three doses using our current schedule and no second year booster would be adequate, but this has not been shown in practice. We could either "be different" and try it, as we have with Hib vaccine, or change our schedule. There is also the issue of the number of injections involved at each visit. Increasing this would receive scant support from the primary care sector, but may become necessary as other new antigens are added to the schedule. The use of combined diphtheria tetanus pertussis and IPV vaccines with (as is now routine in France and Canada) or without Haemophilus influenzae type $b$ in a single injection ${ }^{32}{ }^{36}$ seems to be the 
appropriate way forward if feasible, and UK studies are needed.

There is no doubt that it would be enormously beneficial if all European countries used the same vaccines given at the same times, except where there is a sound epidemiological reason for a difference. Such harmonisation would remove, at a stroke, the need to conduct many studies that are performed simply to show the equivalence of the different schedules before licensing in each country. Until now, familiarly enough, most countries have been willing to discuss a common policy only if it is based on their own established programme. Perhaps the general adoption of eIPV could catalyse the acceptance by Western European countries of a common schedule on which we can all build. A plethora of new and improved antigens (acellular pertussis, hepatitis B, meningococcus group $\mathrm{C}$, and multivalent pneumococcal conjugated capsular polysaccharides, rotavirus, parainfluenza, and respiratory syncytial virus) are currently being introduced, assessed, or developed for primary schedule use. This will involve many clinical trials between now and the time when we can remove polio from the list. This work will be easier if it does not have to be repeated for each of a long list of different schedules.

Whatever decisions are taken, the UK and other developed countries need to maintain or increase their political and financial support for the global eradication of polio using OPV, both in the interests of their own populations and those of the children of the next century worldwide.

1 Cochi SL, Hull HF, Sutter RW, Wilfert CM, Katz SM. of global poliomyelitis eradication. $\mathcal{F}$ Infect Dis 1997;175:S1-3.

2 Eichner M, Dietz K. Eradication of poliomyelitis: when can one be sure that polio virus transmission has been one be sure that polio virus transmission

3 Poliomyelitis outbreak -Albania, 1996. MMWR Morb Mor tal Wkly Rep 1996;45:819-20.

4 Patriarca PA, Sutter RW, Oostvogel PM. Outbreaks of paraPatriarca PA, Sutter RW, Oostvogel PM. Outbreaks of para72 .

5 Paralytic poliomyelitis-United States, 1980-1994 MMWR Morb Mortal Wkly Rep 1997;46:79-83.

6 Salisbury DM, Ramsay ME, White JM, Brown DW. Polio eradication: surveillance implications for the United Kingdom. F Infect Dis 1997;175:S156-9.

7 Minor PD, John A, Ferguson M, Icenogle JP. Antigenic and molecular evolution of the vaccine strain of type 3 poliovirus during the period of excretion by a primary vaccine. $f$ Gen Virol 1986;67:693-706.

8 Kew OM, Nottay BK, Hatch MH, Nakano JH, Obijeski JF. Multiple genetic changes can occur in the oral poliovaccines upon replication in humans. F Gen Virol 1981;56: 337-47.

9 Joce R, Wood D, Brown D, Begg N. Paralytic poliomyelitis in England and Wales, 1985-91. BMF 1992;305:79-82.

10 Nkowane BM, Wassilak SG, Orenstein WA, et al. Vaccineassociated paralytic poliomyelitis. United States: 1973 associated paralytic poliomyelitis. Unite

11 Sabin AB. Is there a need for a change in poliomyelitis immunization policy? Pediatr Infect Dis f 1987;6:887-9.

12 Salk J, van Wezel AL, Stoeckel P, et al. Theoretical and practical considerations in the application of killed poliovirus vaccine for the control of paralytic poliomyelitis. Dev Bio Stand 1981;47:181-98.

13 Paradiso PR. The future of polio immunization in the United States: are we ready for change? Pediatr Infect Dis $f$ 1996;15:645-9.

14 Plotkin SA. Inactivated polio vaccine for the United States: a missed vaccination opportunity. Pediatr Infect Dis $\mathcal{F} 1995$ 14:835-9.

15 Drucker J. Poliomyelitis in France: epidemiology and vaccination status. Pediatr Infect Dis f 1991;10:967-9.

16 Tulchinsky TH. Combined OPV and IPV program in control of poliomyelitis in two endemic areas-a potential tool in the struggle to eradicate poliomyelitis. Public Health Reviews 1993;21:153-6.

17 Ramsay ME, Begg NT, Gandhi J, Brown D. Antibody response and viral excretion after live polio vaccine or a combined schedule of live and inactivated polio vaccines. Pediatr Infect Dis $\mathcal{F}$ 1994;13:1117-21.

18 Faden $\mathrm{H}$, Modlin JF, Thoms ML, McBean AM, Ferdon MB, Ogra PL. Comparative evaluation of immunization with live attenuated and enhanced-potency inactivated trivalent poliovirus vaccines in childhood: systemic and local immune responses. F Infect Dis 1990;162:1291-7.

19 Prevots DR, Sutter RW, Strebel PM, Weibel RE, Cochi SL. Completeness of reporting for paralytic poliomyelitis, United States, 1980 through 1991. Implications for estimating the risk of vaccine-associated disease. Arch Pediatr Adolesc Med 1994;148:479-85.

20 Strebel PM, Sutter RW, Cochi SL, et al. Epidemiology of poliomyelitis in the United States one decade after the last reported case of indigenous wild virus-associated disease. Clin Infect Dis 1992;14:568-79.

21 Recommended childhood immunization schedule-United States, January-December 1997. American Academy of Pediatrics Committee on Infectious Diseases. Pediatrics 1997;99:136-7.

22 Poliomyelitis prevention in the United States: introduction of a sequential vaccination schedule of inactivated poliovirus vaccine followed by oral poliovirus vaccine. Recommendations of the Advisory Committee on Immunization Practices (ACIP). MMWR Morb Mortal Wkly Rep 1997;46: $1-25$

23 Miller MA, Sutter RW, Strebel PM, Hadler SC. Costeffectiveness of incorporating inactivated poliovirus vaccine into the routine childhood immunization schedule. $\mathcal{F A M A}$ 1996;276:967-71

24 Onorato IM, Modlin JF, McBean AM, Thoms ML, Losonsky GA, Bernier RH. Mucosal immunity induced by enhanced-potency inactivated and oral polio vaccines. $\mathcal{f}$ Infect Dis 1991;163:1-6.

25 Ghendon Y, Robertson SE. Interrupting the transmission of wild polioviruses with vaccines: immunological considerations. Bull World Health Organ 1994;72:973-83.

26 Hovi T, Cantell K, Huovilainen A, et al. Outbreak of paralytic poliomyelitis in Finland: widespread circulation of antigenically altered poliovirus type 3 in a vaccinated population. Lancet 1986;i:1427-32.

27 Slater PE, Orenstein WA, Morag A, et al. Poliomyelitis outbreak in Israel in 1988: a report with two commentaries. Lancet 1990;335:1192-5.

28 Conyn van Spaendonck MA, Oostvogel PM, van Loon AM, van Wijngaarden JK, Kromhout D. Circulation of poliovirus during the poliomyelitis outbreak in The Netherlands in 1992-1993. Am f Epidemiol 1996;143:929-35.

29 Schaap GJ, Bijkerk H, Coutinho RA, Kapsenberg JG, van Wezel AL. The spread of wild poliovirus in the well-
vaccinated Netherlands in connection with the 1978 epidemic. Prog Med Virol 1984;29:124-40.

30 Bottiger M, Mellin P, Romanus V, Soderstrom H, Wesslen $\mathrm{T}$, von Zeipel G. Epidemiological events surrounding a paralytic case of poliomyelitis in Sweden. Bull World Health Organ 1979;57:99-103.

31 Chen RT, Hausinger S, Dajani AS, et al. Seroprevalence of antibody against poliovirus in inner-city preschool children. Implications for vaccination policy in the United States. FAMA 1996;275:1639-45.

32 Mallet E, Hoffenbach A, Salomon H, Blondeau C, Fritzell B. Primary immunization with combined, acellular DTaPIPV-Act-HIB vaccine given at 2-3-4 or 2-4-6 months of age [abstract]. 14th meeting of the European Society for Paediatric Infectious Diseases, 1996.

33 Simoes EA, Padmini B, Steinhoff MC, Jadhav M, John TJ. Antibody response of infants to two doses of inactivated poliovirus vaccine of enhanced potency. Am $\mathcal{F}$ Dis Child 1985;139:977-80.

34 Baker JD, Halperin SA, Edwards K, Miller B, Decker M, Stephens D. Antibody response to Bordetella pertussis antigens after immunization with American and Canadian whole-cell vaccines. $\mathcal{F}$ Pediatr 1992;121:523-7.

35 Halperin SA, Langley JM, Eastwood BJ. Effect of inactivated poliovirus vaccine on the antibody response to Bordetella pertussis antigens when combined with diphtheria-pertussis-tetanus vaccine. Clin Infect Dis 1996; 22:59-62.

36 Dagan R, Botujansky C, Watemberg N, et al. Safety and immunogenicity in young infants of Haemophilus b-tetanus protein conjugate vaccine, mixed in the same syringe with diphtheria-tetanus-pertussis-enhanced inactivated poliovirus vaccine. Pediatr Infect Dis $\mathcal{F}$ 1994;13:356-
62 .

\section{Commentary}

At its regional committee meeting in Istanbul on 17 September 1997, the World Health Organisation announced that the eradication of poliomyelitis from the European region may be imminent. Only one case of wild polio was reported from the region in 1997 and this is thought to have been imported from Asia. ${ }^{1}$ Finn and Bell conclude that whatever decisions about polio immunisation in the UK are made, we need to maintain or increase our support for the global eradication of polio using OPV. We concur, and suggest that the best way to 
support this effort is for the UK to continue to use OPV for routine immunisation. We must take a global view-wild polio has not been eradicated.

Outbreaks in Albania ${ }^{2}$ illustrate that cases can occur within a few hours of air travel from the UK. OPV alone has the potential to provide better population immunity and protection of the unvaccinated than IPV. What security do we have that IPV will perform as well as OPV in the UK, particularly when used in an accelerated schedule without a second year booster? The available data are scanty; there are no studies addressing the long term persistence of antibody with this schedule-a policy of "try it and see" is not sound. Only one study has followed infants vaccinated with IPV through to 5 years of age, but doses were given at 2,4 , and 12 months of age; serum immunity was comparable with that after OPV. ${ }^{3}$ More relevant studies are required. The record with OPV in the UK is clear: there has not been a single confirmed case of indigenous wild polio for more than a decade. ${ }^{4}$ Only one relatively small outbreak in an unvaccinated group or a group who had received a poorly immunogenic batch of IPV would exceed the numbers of VAPP predicted to occur over the next few years.

We should also consider how our policy might affect the global agenda. In many less developed countries there is a sizeable middle and upper class whose private doctors are keen to prescribe the vaccines available in the developed world. Demands for IPV may damage the reputation of OPV for those who have no alternative, and all of us are aware of the importance of maintaining public confidence in vaccines.

The harmonisation of vaccine products and schedules in Europe is an important goal. Universal adoption of IPV, however, may not be the best model to launch this, especially as wild polio is a more recent issue in several parts of Europe. Hepatitis B vaccine is arguably a more appropriate starting point as it is already widely used and its implementation would bring us into line with WHO recommendations.

A change of polio vaccine also has cost implications for the UK. IPV is currently about 10 times more expensive to the National Health Service than OPV. Even if the current cost of IPV is halved, this equates to a difference of $£ 8$ million for primary immunisation. Finn and Bell are perhaps too generous in anticipating that manufacturers will ensure that the price of IPV will decrease to be comparable with that of OPV. It is difficult to imagine that IPV will ever be as cheap as OPV. Surely this is an exercise worthy of a formal cost evaluation? It may well be that the money could be better spent elsewhere.

There are also the costs in terms of potential disruptions to the immunisation programme with the introduction of a new vaccine. It is likely that a meningococcal $\mathrm{C}$ conjugate vaccine will be available in the next few years and our energies should be directed towards implementation of this rather than IPV.

The indisputable problem with the continued use of OPV in the UK is that cases of VAPP will occur, albeit at a low rate. An average of about two cases each year were recorded between 1985 and 1994 (Ramsay, personal communication). This is clearly undesirable. A proportion of these cases are preventable; IPV must be given to people who are immunosuppressed or in contact with those who are immunosuppressed. As with all vaccine programmes the risk:benefit ratio becomes less clear as we approach the elimination of disease. A further three years of active surveillance in Europe is required before the region can be officially declared free from polio. Immunisation activities will then continue unabated until all countries have eliminated poliomyelitis. Global certification is hoped for in 2005. We believe OPV remains the vaccine of choice to achieve this goal.

PAUL T HEATH

JENNY M MACLENNAN E RICHARD MOXON Oxford Vaccine Group,

Department of Paediatrics, Fohn Radcliffe Hospital, Oxford OX3 9DU, UK

1 WHO says polio free Europe is imminent. Commun Dis Rep. CDR Wkly 1997;7(38):339.

2 Poliomyelitis outbreak-Albania, 1996. MMWR Morb Mortal Wkly Rep 1996;45:819-20.

3 Faden H, Duffy L, Sun M, Shuff C. Long-term immunity to poliovirus in children immunized with live attenuated and poliovirus in children immunized with live attenuated and
enhanced-potency inactivated trivalent poliovirus vaccines. enhanced-potency inactivated
f Infect Dis 1993; 168:452-4.

F Infect Dis 1993;168:452-4.
4 Salisbury DM, Ramsay ME, White JM, Brown DW. Polio eradication: surveillance implications for the United Kingdom. F Infect Dis 1997;175(suppl 1):S156-9. 